\title{
Study of the hot air drying process of chicken breast by non- invasive techniques
}

\section{Tomas-Egea, J.A..$^{\text {; }}$ Castro-Giraldez, M. ${ }^{a^{*}}$; Colom, R.J. ${ }^{\text {; }}$ Fito, P.J. ${ }^{\text {a }}$}

a Instituto Universitario de Ingeniería de Alimentos para el Desarrollo. Universitat Politècnica de València, Valencia, Spain

${ }^{\mathrm{b}}$ Instituto de Instrumentación para Imagen Molecular, Universitat Politècnica de Valencia, Valencia, Spain.

*marcasgi@upv.es

\begin{abstract}
Food drying is one of the main unit operations for food preservation and it is based on the difference of chemical potential between the product and a fluid with lower chemical potential. The objective of this work was the development of a thermodynamic model of chicken meat drying process using infrared thermography; also the viability of using dielectric spectroscopy as a monitoring system was analyzed. A thermodynamic model has been developed to predict the expansion/contraction phenomena of poultry meat throughout the drying process. Moreover, it was demonstrated that permittivity is a nondestructive method to monitor the evolution of drying process.
\end{abstract}

Keywords: Poultry meat, hot air drying, permittivity, infrared, drying kinetics. 


\section{Introduction}

Food drying is one of the main unit operations to preserve food ${ }^{[1]}$. The process is carried out by the difference of chemical potential between the product and a fluid (hot air) with lower chemical potential. Drying is based on three stages: induction period, period of constant drying speed and period of decreasing drying speed. During the first stage, the water transport occurs due to the difference of chemical potential between the food and the hot air, increasing the temperature of the surface. In the second stage, all the heat is used to evaporate the water, which comes from the inside faster than it evaporates from the surface, therefore, its temperature does not change. After this, the product is dried until the speed with which the water reaches the surface is less than the rate of evaporation and the heat received is used to evaporate the water as well as to heat the product.

Given the importance and extended use of hot air drying in the food industry, to model the behavior of the sample that occurs during dehydration is very useful to optimize the industrial process. A similar thermodynamic model was already reported for hot air drying process of pork meat ${ }^{[2]}$. Several authors have modeled the water transport of other products like red pepper $^{[3]}$, salty pork meat ${ }^{[4,5]}$, apple ${ }^{[6]}$ and sweet potato ${ }^{[7]}$.

The permittivity $\left(\varepsilon^{*}\right)$ is the physical property that describes the interaction between a flux of photons and the medium through which photons circulate. Thus, the analysis of the electrical properties of a flux of photons when it passes through a biological system allow determining the quantity and state of the chemical species of the system. Infrared thermography is a technique that provides information about the heat transfer in meat tissues ${ }^{[8]}$ and consists in the measurement of the infrared radiation emitted by a body surface getting an image of its thermal distribution. Thus, infrared thermography and the permittivity analysis, offer the possibility to analyze the process by a non-destructive way.

The objective of this work is the development of a thermodynamic model of the chicken meat drying process using infrared thermography; also the viability of using dielectric spectroscopy as a monitoring system is analyzed.

\section{Materials and Methods}

\subsection{Raw Material}

The experiments were carried out using boneless broiler breasts (Pectoralis major) obtained from a local supermarket. Cylinders of poultry breast were used for each analysis $(2 \mathrm{~cm}$ of diameter and $1 \mathrm{~cm}$ of height); the cylinders were cut in perpendicular direction to the fibers.

\subsection{Experimental procedure}

Two samples were placed inside the hot air dryer. The drying operation was carried out, for a period of 67 hours, at a temperature of $40^{\circ} \mathrm{C}$ and an air velocity of $1.5 \mathrm{~m} \cdot \mathrm{s}^{-1}$. One of the 
samples was hung from a Mettler Toledo PG503-S balance (precision of $\pm 0.01 \mathrm{~g}$ ) in order to register the mass throughout the drying process. The other sample was placed on the right side of the dryer on a grid. This sample allow controlling continuously the surface temperature. Moreover, two needle electrodes sensor was placed on this sample to measure the permittivity by continuous radiofrequency spectrophotometry. The flat face of both cylindrical samples was located in perpendicular to the drying air. A reference material of known emissivity ( $\varepsilon=0.95$ - Optris $\mathrm{GmbH}$ ) was placed next to the samples. Also, the temperature of the drying air, sample, reference material and environment were measured with K-thermocouples connected to an Agilent multiplexer 34901A (Agilent Technologies, Malaysia) and registered by an Agilent Data Acquisition equipment 34972A (Agilent Technologies, Malaysia). After the drying treatment, the samples were kept in aqualab ${ }^{\circledR}$ disposable sample cups, sealed with parafilm ${ }^{\circledR}$ for further analysis.

Water activity, moisture and volume were measured in fresh samples and also in dried samples. Mass, permittivity and thermal images were registered continuously as was explained above.

\subsection{Infrared measurements}

The infrared analysis was carried out following the method decribed by Traffano-Schiffo et al. (2014) ${ }^{[9]}$, using an infrared camera (Optris $\mathrm{PI}^{\circledR} 160$ thermal imager, Optris GmbH, Berlin, Germany) installed in front of the sample, at an angle of $0^{\circ}$ relative to the plane in which the samples were placed. The camera uses a two-dimensional Focal Plane array with 160x120 pixels, a spectral range of $7.5-13 \mu \mathrm{m}$, resolution of $0.05{ }^{\circ} \mathrm{C}$ and an accuracy of $\pm 2 \%$. The camera covers at a temperature range of -20 to $900^{\circ} \mathrm{C}$. It has a field of vision of $23^{\circ} \mathrm{x} 17^{\circ}$ with a minimum distance of $2 \mathrm{~cm}$. The camera uses Optris PI Connect software (Optris GmbH, Berlin, Germany).

\subsection{Radiofrequency measurements}

The sensor used was developed by the Dielectric Properties Laboratory (Instituto Universitario de Ingenieria de Alimenos para el Desarrollo IuIAD) and the Instituto de Instrumentación para Imagen Molecular (I3M) both belonging to the Universitat Politècnica de València (UPV), Valencia, Spain. It consists of two needle electrodes located on both sides of the cylindrical face of the sample. The sensor is connected to an Agilent 4294A impedance analyzer. The measuring frequency range is from $40 \mathrm{~Hz}$ to $1 \mathrm{MHz}$ and the calibration is performed in open and short-circuit.

\subsection{Physico-chemical measurements}

Water activity was determined with a dew point hygrometer Aqualab ${ }^{\circledR}$, series $3 \mathrm{TE}$, with an accuracy of \pm 0.003 . The mass of the samples was determined with a Mettler Toledo AB304$\mathrm{S}$ balance, with an accuracy of $\pm 0.001 \mathrm{~g}$. The volume was determined with Adobe (C) 
Photoshop (c) CS6 software by image analysis. Moisture was obtained with the ISO 1442 (1997) standard for meat products, drying the samples at $110^{\circ} \mathrm{C}$ and atmospheric pressure until reaching a constant mass.

\section{Results and discussion}

The monitoring of the process with the termographic camera need a correction in the temperature values, that made Traffano Schiffo et al., 2014 ${ }^{[9]}$.

With the evolution of the real temperature obtained by the camera and the physicochemical determinations, it is possible to apply irreversible thermodynamics to model the drying process, in order to analyze the influence of the structure in the water transport during drying. For this purpose, the Gibbs free energy variation (Eq. 1) ${ }^{[10]}$ was applied in the system interface described in figure 1.

$$
d G=-S d T+V d P+F d l+\psi d e+\sum_{i} \mu_{i} d n_{i}
$$

Where: SdT represents the entropic term related to heat fluxes; VdP represents the mechanical energies related to the variation of pressure; Fdl represents the mechanical energies related to the elongation force; and $\psi d e$ represents the effect of the electric field induced by solved ions. The term $\sum_{i} \mu_{i} d n_{i}$ corresponds to the activity term and is the addition of the chemical potentials of the " $i$ " component, being constant the rest of state variables.

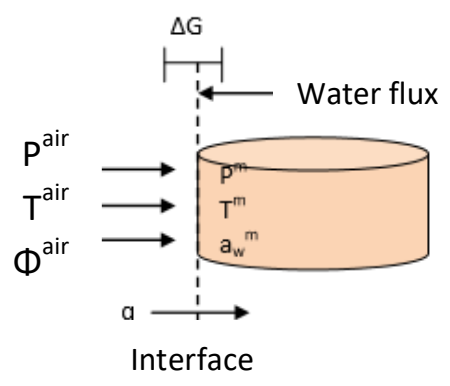

Fig. 1 Diagram of the interface of the chicken sample during drying.

Considering the variation of free energy per mole of water, it is possible to define the extended chemical potential of water according to the equation 2.

$$
\Delta \mu_{w}=\frac{\Delta G}{\Delta n_{w}}
$$

Where: $\Delta \mu_{\mathrm{w}}=$ chemical potential of water $\left(\mathrm{J} \cdot \mathrm{mol}^{-1}\right) ; \Delta \mathrm{G}=$ variation of Gibbs free energy $(\mathrm{J})$; $\Delta \mathrm{n}_{\mathrm{w}}=$ moles of water (mol). 
Combining the last two equations, the equation 3 is obtained. The terms Fdl and $\psi$ de of the equation 1 could be neglected, since the muscle tissue is elastic and the meat only has native ions.

$$
\Delta \mu_{w}=-s_{w}\left(T^{a i r}-T^{m}\right)+v_{w}\left(P^{a i r}-P^{m}\right)+R T^{m} \ln \frac{a_{w}^{m}}{\varphi^{a i r}}
$$

Where: $\mathrm{s}_{\mathrm{w}}=$ partial molar entropy of the water $\left(\mathrm{J} \cdot \mathrm{K}^{-1} \cdot \mathrm{mol}^{-1}\right) ; \mathrm{T}=$ temperature $(\mathrm{K}) ; \mathrm{v}_{\mathrm{w}}=$ partial molar volume of water $\left(\mathrm{m}^{3} \cdot \mathrm{mol}^{-1}\right) ; \mathrm{P}=$ pressure $(\mathrm{Pa}) ; \mathrm{R}=$ constant of ideal gases $(8.314472$ $\left.\mathrm{J} \cdot \mathrm{K}^{-1} \cdot \mathrm{mol}^{-1}\right) ; \mathrm{a}_{\mathrm{w}}=$ water activity of sample; $\varphi=$ relative ambient humidity. Being the superindexes: $\mathrm{m}=$ sample; air $=$ air.

With the temperatures obtained by means of infrared thermography and the evolution of the moisture of the sample, estimated with the variation of mass during the process and the initial moisture, the entropic term can be calculated. For the activities term, a sorption isotherm of poultry was obtained and modeled by $\mathrm{GAB}\left(\mathrm{x}^{0}{ }_{\mathrm{w}}=0.077, \mathrm{C}=420, \mathrm{~K}=0.98\right)$ (Fig. 2). Finally, the term of mechanical energies of the equation 3 (second term) can not be calculated, since the pressure variation of the sample can not be measured.

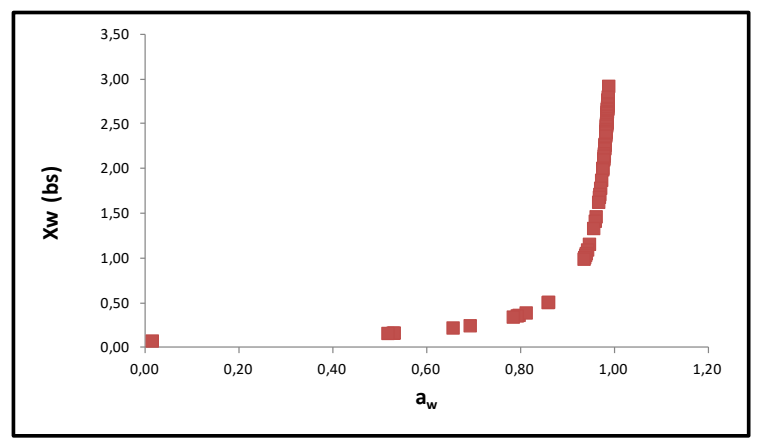

Fig. 2 Poultry sorption isotherm.

According to Onsager's relations ${ }^{[10]}$, the molar water flux is related to its chemical potential, which acts as a driving force for water transport, by means of the phenomenological coefficient (Eq. 4). The phenomenological coefficient is constant in reversible processes, but if the storage of mechanical energy produces irreversible breaks in the medium, the phenomenological coefficient will evolve as a function of the transformation suffered by the tissue.

$$
J_{w}=L_{w} \cdot \Delta \mu_{w}
$$

Where: $\mathrm{J}_{\mathrm{w}}=$ molar flow of water $\left(\mathrm{mol} \cdot \mathrm{s}^{-1} \cdot \mathrm{m}^{-2}\right) ; \mathrm{L}_{\mathrm{w}}=$ phenomenological coefficient $\left(\mathrm{mol}^{2} \cdot \mathrm{J}^{-}\right.$ $\left.{ }^{1} \cdot \mathrm{s}^{-1} \cdot \mathrm{m}^{-2}\right) ; \Delta \mu_{\mathrm{w}}=$ chemical potential of water $\left(\mathrm{J} \cdot \mathrm{mol}^{-1}\right)$. 
In order to obtain the phenomenological coefficient, water flux was estimated as follows (Eq. 5):

$$
J_{w}=\frac{\Delta M_{w}^{m} \cdot M_{0}^{m}}{\Delta t \cdot s^{m} M r_{w}}
$$

Where: $\mathrm{J}_{\mathrm{w}}=$ water flux $\left(\mathrm{mol} \cdot \mathrm{s}^{-1} \cdot \mathrm{m}^{-2}\right) ; \Delta \mathrm{M}_{\mathrm{w}}^{\mathrm{m}}=$ variation of water mass of the sample $(\mathrm{kg}) ; \mathrm{M}_{0}^{\mathrm{m}}$ $=$ initial mass of the sample $(\mathrm{kg}) ; \Delta \mathrm{t}=$ time of the process $(\mathrm{s}), \mathrm{S}^{\mathrm{m}}=$ surface of the sample $\left(\mathrm{m}^{2}\right) ; \mathrm{Mr}_{\mathrm{w}}=$ molecular mass of water $\left(18 \mathrm{~g} \cdot \mathrm{mol}^{-1}\right)$.

As was explained above, it is not possible to calculate the mechanical term of the chemical potential, therefore the chemical potential of water was determined without considering the mechanical terms $\left(\Delta \mu_{w}^{*}\right)$. With the water flux and the chemical potential of water was determined without considering the mechanical terms, the phenomenological coefficient was estimated (Eq. 6).

$$
L_{w}^{*}=\frac{J_{w}}{\Delta \mu_{w}^{*}}
$$

In figure 3 it can be apreciated that there is a linear relation between $L_{w}^{*}$ and $J_{w}$ after 15 minutes of drying. Therefore, following that linear prediction of the phenomenological coefficient it is possible to estimate the mechanical terms with the equation 7 (Fig. 4).

$$
v_{w} \Delta P=\Delta \mu_{w}-\Delta \mu_{w}^{*}
$$

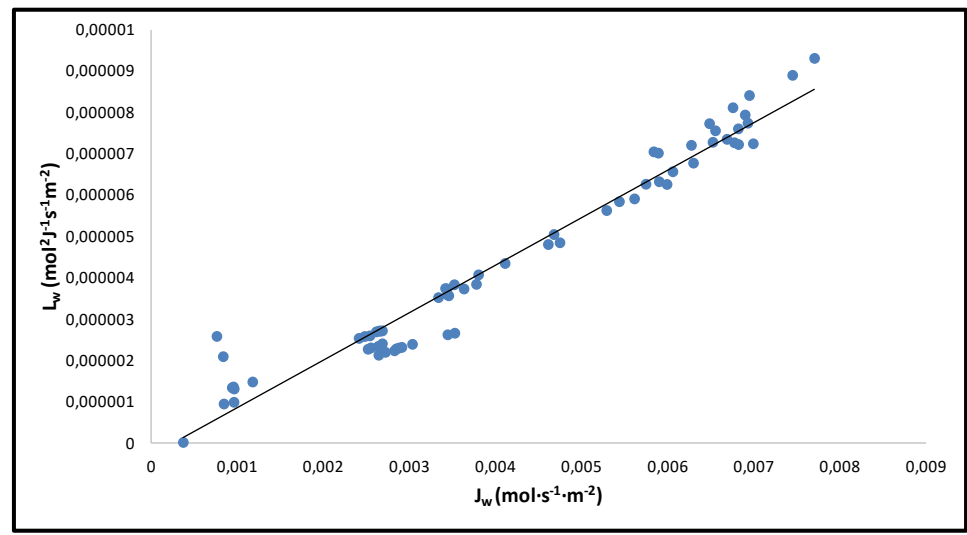

Fig. 3 Relation between the phenomenological coefficient, without considering the mechanical terms, and the water flux from 15 minutes of drying until the end of the treatment.

The evolution of the mechanical energy indicates that there is an expansion of the tissue during the first 400 minutes, followed by a contraction associated with the remaining functioning of the muscle from that time (Fig. 4). 


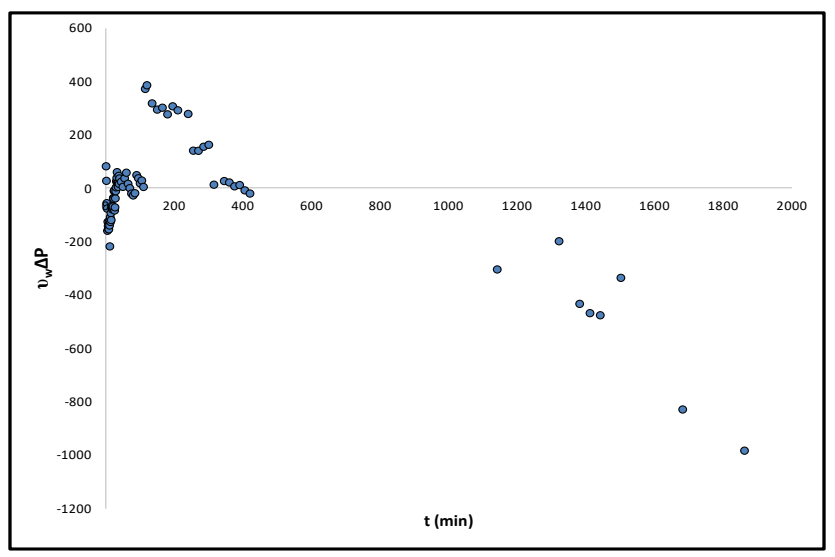

Fig. 4 Evolution of mechanical energy, developed from the thermodynamic model, throughout the drying treatment.

The permittivity in radiofrequency was measured throughout the drying treatment. It was found a linear relation between the dielectric constant in $\alpha$-dispersion $(40 \mathrm{~Hz}$ ) and the number of water molecules when the sample still presents liquid phase, since this dispersion is fundamentally related to the mobility of the ions dissolved in meat liquid phase. With the drying time, the content of the liquid phase begins to decrease (samples with adsorbed water only) and also the mobility of the ions. The value of the dielectric constant in this dispersion becomes practically null.

\section{Conclusions}

It has been demonstrated that infrared thermography is a good technique to monitor the process of drying in poultry meat, providing information of heat transfer in biological tissues, being possible to obtain the evolution of the emissivity of the meat. Thus, with this technique, it is possible to non-invasively obtain the surface temperature of the sample during the treatment.

A thermodynamic model has been developed to predict the expansion/contraction phenomena of poultry meat throughout the drying process, as well as to evaluate the driving forces of the drying process.

Finally, a direct relation between the permittivity in $\alpha$-dispersion with regard to the number of water molecules has been demonstrated. It can be concluded that the permittivity is a nondestructive and rapid method to monitor the evolution of the drying process.

\section{Acknowledgements}

The authors acknowledge the financial support from: the Spanish Ministerio de Economía, Industria y Competitividad, Programa Estatal de I+D+i orientada a los Retos de la Sociedad 
AGL2016-80643-R, Agencia Estatal de Investigación (AEI) and Fondo Europeo de Desarrollo Regional (FEDER). Juan Ángel Tomás Egea wants to thank the FPI Predoctoral Program of the Universidad Politécnica de Valencia for its support.

\section{References}

[1] Ratti, C.; Hot air and freeze-drying of high-value foods: a review. Journal of Food Engineering 2001, 49, 311-319.

[2] Doymaz, I.; Pala, M. Hot-air drying characteristics of red pepper. Journal of Food Engineering 2002, 55(4), 331-335.

[3] Gou, P.; Comaposada, J.; Arnau, J. Meat pH and meat fibre direction effects on moisture diffusivity in salted ham muscles dried at $5^{\circ} \mathrm{C}$. Meat science 2002, 61(1), 25-31.

[4] Clemente, G.; Bon, J.; Sanjuán, N.; Mulet, A. Drying modelling of defrosted pork meat under forced convection conditions. Meat science 2011, 88(3), 374-378.

[5] Wang, Z.; Sun, J.; Liao, X.; Chen, F.; Zhao, G.; Wu, J.; Hu, X. Mathematical modeling on hot air drying of thin layer apple pomace. Food research international 2007, 40, 3946.

[6] Diamante, L.M.; Munro, P.A. Mathematical modelling of hot air drying of sweet potato slices. Food science and technology 1991, 26, 99-109.

[7] Traffano-Schiffo, M.V.; Castro-Giraldez, M.; Fito P.J.; Balaguer, N. Thermodynamic model of meat drying by infrarred thermography. Journal of Food Engineering 2014, 128(0), 103-110.

[8] Workmaster B. A.; Palta, J. P.; Wisniewski, M. Ice nucleation and propagation in cranberry uprights and fruit using infrared video thermography. Journal of the American Society for Horticultural Science 1999, 124, 619.

[9] Traffano-Schiffo, M. V.; Castro-Giráldez, M.; Fito, P. J.; Balaguer, N. Thermodynamic model of meat drying by infrarred thermography. Journal of Food Engineering 2014, 128, 103-110.

[10] Castro-Giráldez, M.; Fito, P.J.; Fito P. Non-equilibrium thermodynamic approach to analyze the pork meat (Longissimus dorsi) salting process. Journal of Food Engineering 2010, 99(1), 24-30. 